\title{
Delayed Endovascular Coil Extrusion following Internal Carotid Artery Embolization
}

\author{
Matthew Dedmon ${ }^{1,2}$ Josh Meier ${ }^{1,2}$ Kyle Chambers ${ }^{1,2}$ Aaron Remenschneider ${ }^{1,2}$ Brijesh Mehta $^{3}$ \\ Derrick Lin ${ }^{1,2}$ Albert J. Yoo ${ }^{3}$ William Curry ${ }^{4}$ Stacey Gray ${ }^{1,2}$
}

\footnotetext{
${ }^{1}$ Department of Otolaryngology - Head \& Neck Surgery, Massachusetts Eye \& Ear Infirmary, Boston, Massachusetts, United States

2 Department of Otology \& Laryngology, Harvard Medical School, Boston, Massachusetts, United States

${ }^{3}$ Department of Radiology, Massachusetts General Hospital, Harvard Medical School, Boston, Massachusetts, United States

${ }^{4}$ Department of Neurosurgery, Massachusetts General Hospital, Harvard Medical School, Boston, Massachusetts, United States
}

\author{
Address for correspondence Dr. Matthew Dedmon, MD, PhD, \\ Department of Otolaryngology, Massachusetts Eye and Ear Infirmary, \\ 243 Charles Street, Boston, MA 02114, United States \\ (e-mail: matthew_dedmon@meei.harvard.edu).
}

J Neurol Surg Rep 2014;75:e255-e258.

\begin{abstract}
Keywords

- endoscopic sinus surgery

- endoscopic endonasal skull base surgery

- internal carotid artery injury

- endovascular embolization

Internal carotid artery injury is a rare and devastating complication of endoscopic sinus and skull base surgery that has an associated mortality rate of $15 \%$. This case describes a patient who developed massive epistaxis following routine sinus surgery and was eventually diagnosed with a pseudoaneurysm of the cavernous internal carotid artery. Endovascular coiling and Onyx (Covidien, Irvine, California, United States) liquid embolization were ultimately used to completely occlude the internal carotid artery with resolution of bleeding; however, the patient had an unexpected late complication of coil extrusion through the pseudoaneurysm sac into the sphenoid sinus and nasal cavity. The endoscopic skull base team safely excised the coils endoscopically without recurrent bleeding. We describe the multidisciplinary operative management of this case of endovascular coil extrusion to increase awareness of this potentially life-threatening complication.
\end{abstract}

\section{Introduction}

Internal carotid artery injury is arguably the most feared complication of endoscopic sinus and skull base surgery. Although typically heralded by massive intraoperative hemorrhage, injuries to the internal carotid artery may go undetected and result in pseudoaneurysm formation with delayed bleeding. ${ }^{1}$ Additional complications such as spasm, embolic events, thrombosis, or caroticocavernous fistula formation are also possible. The internal carotid artery is potentially vulnerable during instrumentation of the sphenoid sinus because the bony covering can be $<0.5 \mathrm{~mm}$ thick. ${ }^{2}$ Moreover, the lateral wall of the sphenoid sinus overlying the carotid artery has been noted to be dehiscent in as many as $22 \%$ of cases. ${ }^{2}$ With these anatomic factors in mind, it is fortunate that carotid artery injury is an exceedingly rare complication of routine endoscopic sinus surgery, with an estimated incidence far $<1 \%{ }^{1}$ However, more aggressive endoscopic endonasal skull base resections have been associated with an increased risk of carotid artery injury ranging from 1 to $9 \%{ }^{1,3}$ Primary management strategies for iatrogenic carotid injury during routine endoscopic sinus surgery include nasal packing followed by emergent angiography to identify the source of bleeding. Endovascular coiling and arterial embolization methods are often used, and complete occlusion of the internal carotid artery may be required for control of bleeding. ${ }^{4}$ Unfortunately, delayed treatment complications such as endovascular coil extrusion may occur after carotid embolization and create concern for further episodes of life-threatening bleeding.
License terms

Stuttgart · New York

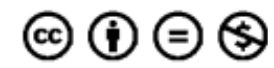

(C) 2014 Georg Thieme Verlag KC
May 7, 2014

accepted

June 11,2014

published online

November 12, 2014
DOI http://dx.doi.org/

10.1055/s-0034-1387193. ISSN 2193-6358. 

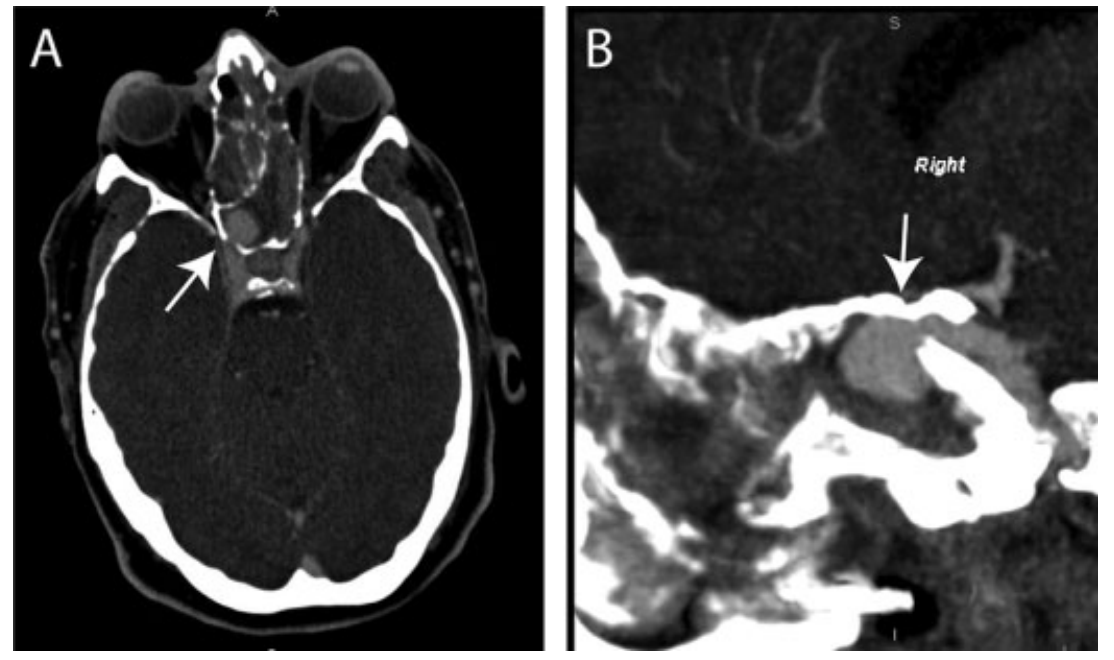

Fig. 1 Computed tomography angiogram revealed a 13-mm pseudoaneurysm arising from the right cavernous internal carotid artery projecting into the right sphenoid sinus. (A) Axial. (B) Sagittal. Arrows point to the pseudoaneurysm.

\section{Case Report}

A 44-year-old man experienced several episodes of massive epistaxis following routine endoscopic sinus surgery at an outside facility. Bilateral sphenopalatine and facial artery embolization was performed at that facility, but he developed another episode of significant nasal bleeding immediately after his nasal packing was removed, and he was transferred to our institution. Nasal packing was replaced at the outside facility that controlled the epistaxis. He was critically ill and required intubation for airway protection, transfusion of 3 units of packed red blood cells, as well as vasopressor therapy prior to transfer. A computed tomography (CT) angiogram on his arrival demonstrated a 13-mm pseudoaneurysm arising from the right cavernous internal carotid artery projecting into the superior sphenoid sinus through a focally dehiscent carotid canal ( $\mathbf{- F i g}$. 1A, B). The patient was taken to the neurointerventional radiology suite, and platinum endovascular coils were deployed to obliterate the pseudoaneurysm (-Fig. 2A-C). Revision endovascular coiling was performed 1 week later following additional epistaxis; angiography at that time identified a recurrent $8-\mathrm{mm}$ pseudoaneurysm. Two days later the patient experienced massive epistaxis with blood loss $>1500 \mathrm{~mL}$. The internal carotid artery was subsequently completely occluded with balloon-assisted coiling and Onyx (Covidien, Irvine, California, United States) liquid embolic agent. The patient had adequate collateral blood flow to the right cerebral hemisphere and retained normal neurologic function after the procedure. He did very well with no further bleeding events after carotid occlusion.

As a consequence of his original sinus surgery and longterm nasal packing, the patient developed a large nasal septal perforation and significant nasal crusting. He was closely followed by the otolaryngology service with frequent sinonasal debridements. Two months following complete internal carotid artery occlusion, the patient was noted to have a single coil emerging from the right sphenoid ostium extending into the nasal cavity on endoscopic examination ( - Fig. 3A). The coil subsequently extruded $6 \mathrm{~cm}$ outside the nasal cavity after the patient blew his nose; the preoperative CT scan demonstrated the aberrant coil ( - Fig. 3B). The patient was taken emergently to the operating suite for angiography, endoscopic surgical exploration, and coil extraction.
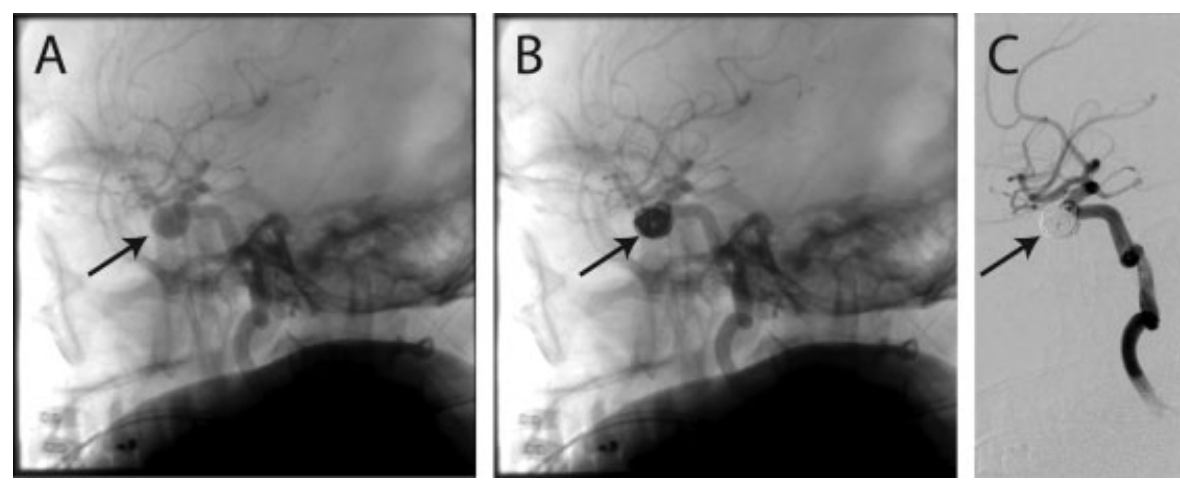

Fig. 2 (A) A $10 \times 12-m m$ pseudoaneurysm of the right cavernous internal carotid artery was seen on angiography. Platinum coils were deployed within the pseudoaneurysm (B) to achieve successful obliteration (C). Arrows point to the pseudoaneurysm. 

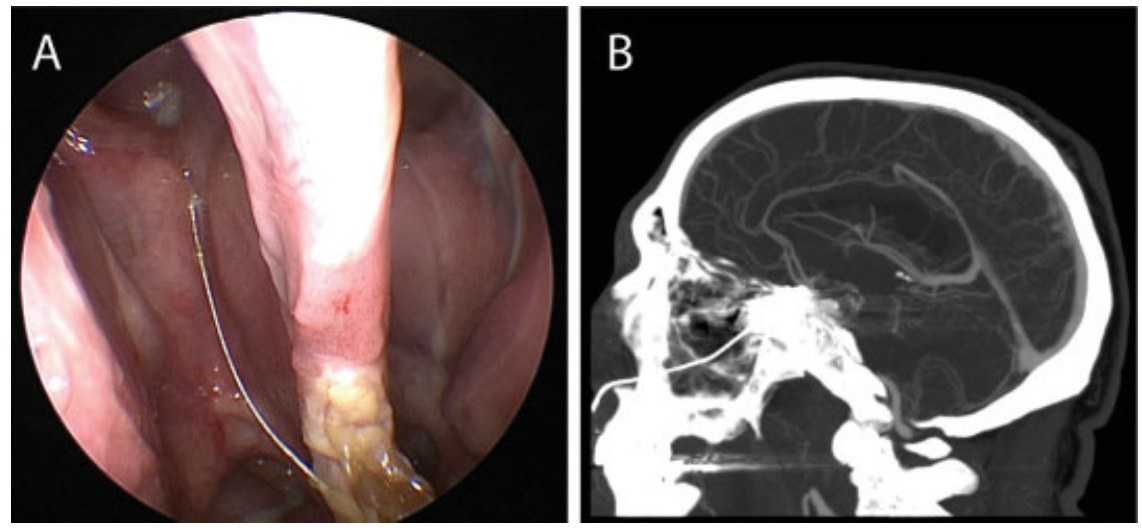

Fig. 3 (A) Nasal endoscopy showed a single coil emerging from the right sphenoid ostium extending into the nasal cavity. (B) Computed tomography scan demonstrating the aberrant coil.

An intraoperative angiogram demonstrated stable occlusion of the right internal carotid artery, and the vascular catheters were left in place to facilitate deployment of an intracranial balloon in the event of massive hemorrhage during the endoscopic exploration. A large bilateral sphenoidotomy with removal of the residual posterior nasal septum and intersphenoid septum was performed to improve exposure to the right sphenoid sinus. The right sphenoid sinus was filled with an extruded coil mass surrounded by purulent discharge and granulation tissue (-Fig. 4). A two-surgeon endoscopic skull base team, including otolaryngology and neurosurgery, meticulously dissected and divided the aberrant coils within the sphenoid sinus and removed them. Extruded Onyx embolic material was also encountered within the sphenoid sinus and was debulked. Some coils embedded in the Onyx matrix were communicating directly with the internal carotid artery through the carotid canal defect. These coils were trimmed at the level of the defect, and the embedded portions were left in place in the carotid artery.

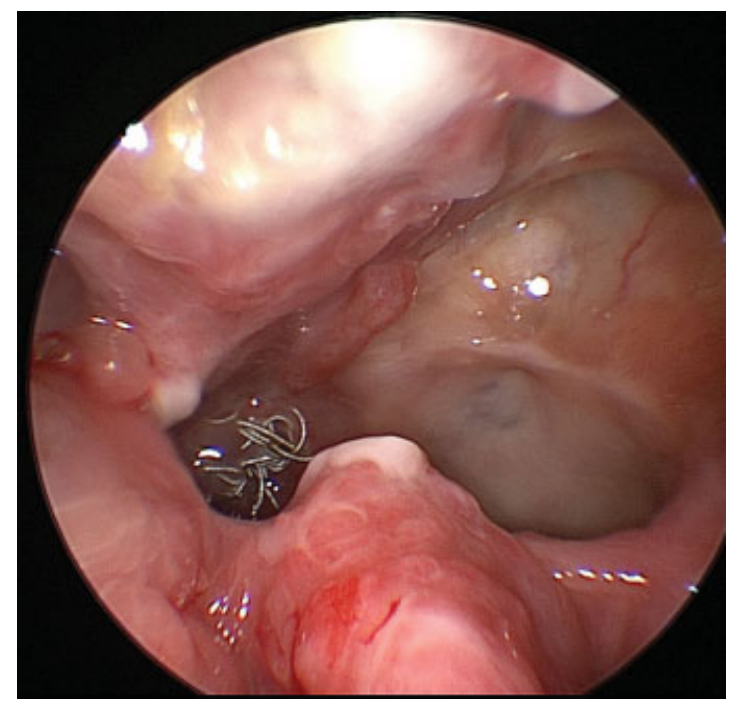

Fig. 4 Extruding coils within the right sphenoid sinus at the time of surgery.
No significant bleeding was encountered during the procedure. The patient had an uneventful postoperative course and was subsequently discharged with no further bleeding events.

In the past year since carotid sacrifice, the patient has had no significant episodes of epistaxis. Follow-up angiography confirmed continued complete occlusion of the right internal carotid artery. Four months later the patient experienced another instance of coil extrusion following nose blowing. He underwent reexcision of several small displaced coils from the interior of the sphenoid sinus in the operating room without complication. Office endoscopy continues to be performed routinely to monitor for evidence of recurrent coil extrusion from the obliterated carotid.

\section{Discussion}

Internal carotid artery injury following routine endoscopic sinus surgery is an infrequent event, with only several reported cases in the literature. ${ }^{1}$ However, suspicion for this devastating complication must remain high in the setting of intraoperative and postoperative epistaxis due to the high associated mortality of carotid injury. CT and traditional angiography remain critical components in the evaluation of suspected carotid artery injuries, particularly in the identification of a delayed complication such as pseudoaneurysm formation. Endovascular coiling and embolization may subsequently be required to occlude either the pseudoaneurysm or the carotid artery itself to control bleeding. The walls of an arterial pseudoaneurysm are inherently unstable, and metallic coils have the potential to dislodge and escape the confines of the pseudoaneurysm. ${ }^{5}$ In this anatomic area, extrusion can occur into the sinonasal cavity, as demonstrated in this case, or possibly into the intracranial space.

Extruded coils have the potential to destabilize the prior arterial embolization, and they should be managed emergently in the operating room. A multidisciplinary approach is favored, with the involvement of otolaryngologists, neurosurgeons, as well as neurointerventional radiologists ready to perform intraoperative angiography and endovascular procedures should hemorrhage occur. In this case, the presence 
of Onyx liquid embolic matrix in the internal carotid artery and pseudoaneurysm likely decreased the chance of further hemorrhage following coil extrusion. With this in mind, the decision was made to leave the remaining embedded coils undisturbed rather than risk destabilization of the occlusive matrix. Although the patient had a subsequent coil extrusion event requiring operative management, the Onyx matrix has remained in place within the carotid artery, and no further bleeding events have occurred.

\section{Conclusion}

We have described a case of endovascular coil extrusion from an internal carotid artery pseudoaneurysm that developed secondary to traumatic injury during routine endoscopic sinus surgery. This complication occurred several months following endovascular control of the original internal carotid artery injury, and patients undergoing such embolization procedures should therefore be closely monitored for this complication. Although exceedingly rare in endoscopic sinus surgery, injuries to the internal carotid artery are more frequent in endoscopic transsphenoidal approaches to the pituitary and in extended endonasal skull base approaches, with estimated incidences of 1 to $9 \% .^{1,3}$ With the advent of endonasal techniques that facilitate more aggressive sinus and skull base resections, surgeon familiarity with carotid injuries and management will be critical because such complications have the potential to become more frequent.
Financial Disclosures

The authors have nothing to disclose.

Note

This case report was presented as a poster at the 2014 North American Skull Base Society Meeting.

\section{Conflict of Interest}

The authors having nothing to disclose.

\section{References}

1 Valentine R, Wormald PJ. Carotid artery injury after endonasal surgery. Otolaryngol Clin North Am 2011;44(5):1059-1079

2 Fujii K, Chambers SM, Rhoton AL Jr. Neurovascular relationships of the sphenoid sinus. A microsurgical study. J Neurosurg 1979; 50(1):31-39

3 Gardner P, Tormenti M, Pant H, Fernandez-Miranda JC, Snyderman $\mathrm{CH}$, Horowitz MB. Carotid artery injury during endoscopic endonasal skull base surgery: incidence and outcomes. Neurosurgery 2013;73 (2 Suppl Operative): 261-269;discussion 269-270

4 Koitschev A, Simon C, Löwenheim H, Naegele T, Ernemann U. Management and outcome after internal carotid artery laceration during surgery of the paranasal sinuses. Acta Otolaryngol 2006; 126(7):730-738

5 Chen D, Concus AP, Halbach VV, Cheung SW. Epistaxis originating from traumatic pseudoaneurysm of the internal carotid artery: diagnosis and endovascular therapy. Laryngoscope 1998;108(3): 326-331 